\title{
Repeatability of Automated FIB Prepared TEM Samples with Low keV Cleaning
}

\author{
Michael H. Moriarty, Jeff E. Blackwood, Jason H. Arjavac
}

FEI Company, 5350 NE Dawson Creek Drive, Hillsboro, Oregon 97124 USA

TEM analysis is critical for answering many of today's most interesting problems. Focused Ion Beam (FIB) processing is the standard method for efficiently preparing high-quality TEM samples in many laboratories. For an overview of the techniques and the motivations for using FIB in TEM sample preparation, see [1]. Automated FIB processes can produce TEM samples with a mean thickness less than $60 \mathrm{~nm}$ and a $1 \sigma$ thickness variation less than $5 \mathrm{~nm}$ at the completion of low $\mathrm{keV}$ FIB cleaning. For analyses or investigations that require TEM images of multiple similar structures, pattern recognition techniques allow TEM samples to be extracted from features of interest at a rate of 3 to 4 samples per hour with $1 \sigma$ position repeatability better than $8 \mathrm{~nm}$.

The solutions discussed here were developed for full wafer production line process monitoring in the data storage and semiconductor industries, where a manufacturing facility may be required to produce and measure tens of thousands of STEM images in the course of a year to maintain product yield [2][3]. These solutions have also been applied to use cases where there are different sites of interest on each wafer, such as failure analysis, by replacing a pattern recognition step with an interface that allows an operator to identify and queue sites of interest via mouse clicks [4]. The process described here performs all sample preparation steps (including final $5 \mathrm{keV}$ cleaning) while the sample is still on the bulk substrate. A separate glass rod liftout tool is then used to transfer the completed samples from the substrate to a TEM grid.

The data presented in Table 1 was measured in dual beam systems on TEM samples produced in those same systems. These tools contain a FIB column that can be tilted relative to the sample, a SEM column, and a stage capable of holding $300 \mathrm{~mm}$ diameter semiconductor wafers. [5] TEM samples were produced and measured in sets containing between 4 and 21 sites in order to calculate statistics on sample variation. The process landing error was measured using top-down FIB images. The position error at a site is calculated as a sum of measurements from two diagnostic images captured during processing of the site. See Figure 1a for an example of this measurement. Row 5 in Table 1 shows the $1 \sigma$ variation measured from each set. To obtain a thickness measurement from each TEM sample, the surfaces of the lamella were coated with a secondary-electron induced Tungsten deposition and crossed-sectioned using the FIB. The Silicon thickness between the two coated surfaces was measured at a distance $250 \mathrm{~nm}$ below the original wafer surface as shown in Figure 1c. Row 10 in Table 1 shows the mean thickness of each set that was treated with $5 \mathrm{keV}$ FIB cleaning. Row 11 shows the $1 \sigma$ variation of the measurements. Rows 7 and 8 show the same dimensions from sets of control sites processed in parallel and halted prior to the $5 \mathrm{keV}$ FIB cleaning step.

By applying a root sum square error tolerance calculation to these measurements, one can determine the minimum feature size that this TEM sample preparation method can reliably capture. For the drift-corrected $5 \mathrm{keV}$ cleaned process, the placement and thickness deviation indicates that the process will successfully capture a $70 \mathrm{~nm}$ feature with a greater than $90 \%$ success rate.

It has been demonstrated that preparing samples with a $30 \mathrm{keV} \mathrm{FIB} \mathrm{can} \mathrm{cause} \mathrm{sidewall} \mathrm{damage}$ to a depth of approximately $21 \mathrm{~nm}$ from the milled surface. Cleaning the sample faces with $5 \mathrm{keV}$ Gallium ions can remove the initial damage layer and leave less than $5 \mathrm{~nm}$ of damage on each sample face. [6] This is the motivation for removing a total of $45-50 \mathrm{~nm}$ (half from each sample 
face) from the sample using the $5 \mathrm{keV}$ FIB. Compare the difference between rows 7 and 10 in Table 1. To avoid the potential issue of sputtering material from the bulk onto the backside of a sample during $5 \mathrm{keV}$ cleaning, the system used an improved power supply and optics that allow the system to precisely raster the low $\mathrm{keV}$ beam on the sample face without overlapping bulk material.

These automated processing are yielding high-quality, $5 \mathrm{keV}$ FIB cleaned TEM samples with high robustness suitable for analyzing $70 \mathrm{~nm}$ features of interest.

\section{References}

[1] Mayer et al., TEM Sample Preparation and FIB Induced Damage, MRS Bulletin 32 (2007) 400.

[2] H. Wang, J. Fang, J. Arjavac, R. Kellner, "Scanning Transmission Electron Microscopy for Critical Dimension Monitoring in Wafer Manufacturing," Microsc. Today (Jan 2008) 24.

[3] B. Rijpers, D. Verkleij, E. Langer, "Automated TEM sample preparation on wafer level for metrology and process control," Proc. Int. Sym. for Testing and Failure Analy. (2007) 107.

[4] J.J. Demarest, Z. DeSouza, "Startup of a Dual Beam FIB for Automatic STEM Sample Preparation”, Proc. Int. Sym. for Testing and Failure Analy. (2009) 334.

[5] This particular platforms used were FEI's Helios 1200 and Certus P2 DualBeam systems.

[6] L.A. Giannuzzi, R. Geurts, J. Ringnalda, Microsc. Microanal. 11 suppl. 2 (2005) 828.
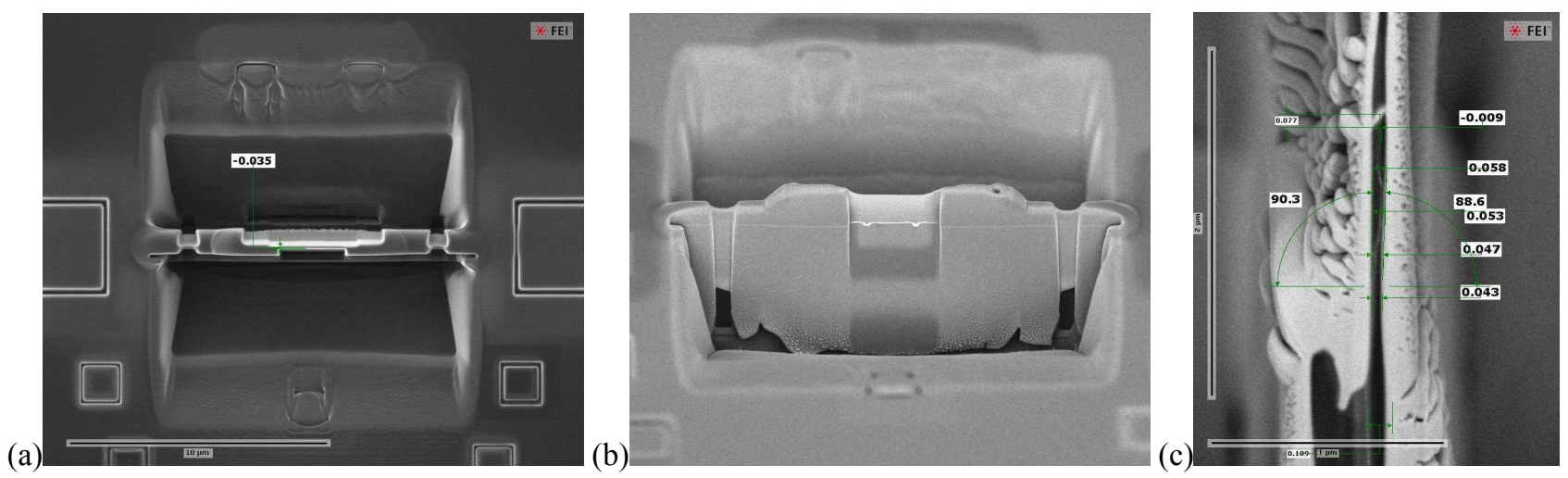

FIG. 1. (a) Top-down FIB images used to measure process placement relative to target structure (b) SEM images of completed TEM sample ready for ex-situ transfer to TEM grid (c) SEM image of cross-sectioned TEM sample.

Original sample (dark) is coated on both faces by Tungsten (bright) and measures $58 \mathrm{~nm}$ thick at depth of interest.

TABLE 1. Measurements gauging the repeatability of TEM sample preparation methods across multiple tools and days.

\begin{tabular}{|c|c|c|c|c|c|c|c|c|c|c|}
\hline \multicolumn{11}{|c|}{ Test Condition Information } \\
\hline 1 & System & Tool 1 & Tool 1 & Tool 1 & Tool 1 & Tool 2 & Tool 2 & Tool 3 & Tool 3 & Tool 3 \\
\hline 2 & Date & Day 1 & Day 2 & Day 3 & Day 4 & Day 1 & Day 2 & Day 1 & Day 2 & Day 3 \\
\hline \multirow[t]{2}{*}{3} & Drift Control & Disabled & Disabled & Disabled & Enabled & Enabled & Enabled & Disabled & Disabled & Disabled \\
\hline & \multicolumn{10}{|l|}{ Process Placement } \\
\hline 4 & Number of Sites Measured & 57 & 20 & 25 & 47 & 0 & 19 & 5 & 5 & 4 \\
\hline 5 & Variation $(\mathrm{nm} 1 \sigma)$ & 20.0 & 22.6 & 25.8 & 7.6 & $\mathrm{~N} / \mathrm{A}$ & 6.0 & $8.4^{*}$ & $7.0^{\star}$ & $2.6^{*}$ \\
\hline \multicolumn{11}{|c|}{ Sample Thickness - Control Sites Arrested and Measured Prior to $5 \mathrm{keV}$ FIB Cleaning } \\
\hline 6 & Number of Sites Measured & 20 & 0 & 16 & 21 & 20 & 5 & 5 & 5 & 4 \\
\hline 7 & Mean Thickness (nm) & 99.5 & $\mathrm{~N} / \mathrm{A}$ & 99.0 & 106.3 & 100.9 & 101.7 & 92.1 & 99.4 & 97.1 \\
\hline 8 & Variation $(\mathrm{nm} 1 \sigma)$ & 1.7 & $\mathrm{~N} / \mathrm{A}$ & 2.5 & 2.8 & 1.8 & 1.6 & 2.9 & 1.6 & 5.0 \\
\hline \multicolumn{11}{|c|}{ Sample Thickness - Sites Measured after Completion with 5 keV FIB Cleaning } \\
\hline 9 & Number of Sites Measured & 20 & 18 & 12 & 18 & 20 & 7 & 0 & 0 & 0 \\
\hline 10 & Mean Thickness (nm) & 51.4 & 55.7 & 57.6 & 57.0 & 52.2 & 58.7 & $\mathrm{~N} / \mathrm{A}$ & $\mathrm{N} / \mathrm{A}$ & $\mathrm{N} / \mathrm{A}$ \\
\hline 11 & Variation $(\mathrm{nm} 1 \sigma)$ & 10.5 & 3.5 & 5.4 & 3.3 & 3.8 & 3.1 & $\mathrm{~N} / \mathrm{A}$ & $\mathrm{N} / \mathrm{A}$ & $\mathrm{N} / \mathrm{A}$ \\
\hline
\end{tabular}

* On Tool 3, only the final placement to reference feature offset was measured. Based on a model of the placement error mechanism, the measured

standard deviation value was mulitiplied by $\sqrt{2}$ to meaninfully represent the same metric as the other two tools. 